\title{
Review of Matthew Adler's Well-being and fair distribution: beyond cost-benefit analysis. Oxford: Oxford University Press, 2011, 656 pp.
}

\author{
EFTHYMIOS ATHANASIOU \\ New Economic School, Moscow
}

Matthew Adler develops and defends a particular view on how the appraisal of social policies should be performed and in doing so offers a new and refreshing take on the subject of fair distribution.

Adler argues for prioritarianism, which, broadly speaking, acknowledges that inequality in the distribution of well being across individuals constitutes an important factor in collective decisionmaking. Adler does not contribute a novel prioritarian social welfare function. He provides a more thorough justification for one among those the literature has already identified (see Moulin 1988). In pursuit of this agenda, Adler takes us through all the stages of the construction of his prioritarian argument, from the foundational underpinnings to its policy implications. With this book, Adler accomplishes the daunting task of merging both the philosopher's and the economist's perspectives on the issues at hand. His exposition, as well as his line of reasoning, is enhanced by the fact that each topic is addressed from multiple angles.

The argument of the book develops against the backdrop of the fundamental exercise that underlies welfare analysis. Let $N=\{1,2, \ldots, n\}$ denote the set of individuals and $X=\{x, y, \ldots, z\}$ denote the set of outcomes. Moreover, for each individual $i \in N$, let $u_{i}: X \rightarrow R$ be an index of individual well being at each outcome $x \in X$. Adopting this premise, one is confronted with the question of what constitutes the appropriate criterion for ranking outcomes from a collective point of view.

The book puts forward a particular prioritarian view on the matter of how a measure of social welfare should be constructed. A prioritarian social choice function bears the following form. Let $g: R \rightarrow R$ be a nondecreasing and concave function. For each pair of outcomes $x, y \in X, x$ is socially at least as good as $y$ if and only if

$$
\sum_{i \in N} g\left[u_{i}(x)\right] \geq \sum_{i \in N} g\left[u_{i}(y)\right]
$$


This broad family of social welfare functions exhibits aversion to inequality in the distribution of well being that each outcome induces. The degree of aversion varies with the degree of concavity of the function $g$. At the two extremes lie utilitarianism, which exhibits no aversion to inequality, and lexi-min, which is infinitely averse to inequality. Adler rejects these two extremes and provides a rationale for intermediate solutions.

This simple framing of the problem reveals the set of issues that one needs to tackle in order to argue in favor of prioritarianism. Adler meticulously reviews each of them in an effort to build a sound and comprehensive argument. First, one needs to accept a welfarist premise: individual indices of well being are the appropriate basis on which to found a criterion of social choice. Adler discusses the doctrine of welfarism in chapter 1 , where he juxtaposes its requisites against competing theories, while providing an account of its philosophical foundations and moral implications.

Second, one needs to take a stance on the issue of the informational basis of the aggregation exercise. What information is conveyed by the individual indices of well being that a social planner may use to construct a social welfare function? In chapter 3, Adler promotes the view that such indices convey cardinal information and are, moreover, amenable to interpersonal comparisons. Although the case for prioritarianism could be promoted under alternative hypotheses, Adler's particular proposal requires this much information to be conveyed by individual behavior. He offers his own take on what constitutes a foundational theory that lends credence to the assumptions of cardinality and interpersonal comparability.

We are left then with one final question. Departing from a welfarist premise and operating on the information that the measure of individual well being conveys, how does one settle on a particular class of $g$ functions? In chapter 5, Adler analyses the properties that the exercise of aggregation of individual utilities should abide by. He puts forward a list of axioms. These are properties of the social welfare function. ${ }^{1}$ They impose on it adherence to certain principles whose validity may be ascertained in non-suspect time, that is without knowledge of the actual realization of individual preferences.

\footnotetext{
${ }^{1}$ A nice complement to this chapter is Herve Moulin's (1988) review of the formal results on which Adler bases his axiomatic argument.
} 
The Pigou-Dalton axiom stands out as a fundamental principle that underlies the prioritarian perspective. If a distribution of utilities induced by some outcome $x \in X$ can be derived by another distribution of utilities induced by an outcome $y \in X$ by means of a hypothetical transfer in utilities between two individuals that preserves the sum of utilities and does not alter the individuals' relative rank in the distribution, then any social welfare function that satisfies the axiom should deem outcome $x$ as socially better than outcome $y$.

Along with Pigou-Dalton, Adler puts forward a list of axioms and justifies their importance. He makes a good case for each of these, both on moral as well as practical grounds. These axioms uniquely determine a parametric class of social welfare functions. Each member of this family corresponds to a different degree of inequality aversion. Utilitarianism is rejected because Adler's version of the Pigou-Dalton principle does not allow for it. The lexi-min is rejected because it violates continuity, a formal property to which Adler attributes ethical content. What remains is unambiguously prioritarian.

There remains the issue of the degree of inequality aversion, the intensity of prioritarianism. Adler finds that this cannot be resolved ex ante. Instead, the social planner needs to become aware of individual preferences before being in a position to employ a fully specified social welfare function. Adler proposes a series of thought experiments performed by individuals that aim at eliciting their degree of inequality aversion. Here too, cardinality and the interpersonal comparability of utilities are crucial for such an approach to work.

Although the preceding account captures what in my view constitutes the backbone of Adler's book, it should be noted that he also takes up issues that are peripheral to his principal concern. In chapter 4 he discusses the foundations of utility theory and surveys different views on the matter. In chapter 6 he defends the idea that the aggregation problem should be framed in terms of entire lifetimes (i.e., utilities are defined over outcomes that encompass the entire span of individual lives).

In chapter 7 he embarks on the task of defending expected utility theory. The goal being not merely to demonstrate that prioritarianism is compatible with uncertainty, but, more importantly, to defend the premise that a theory of utility can be derived from well-behaved individual preferences over uncertain outcomes. Finally, in chapter 8, which concludes the book, Adler contrasts the implications of the 
social-welfare approach with those of competing approaches such as cost-benefit analysis, and alludes to possible extensions of his findings.

The fundamental problem that lies at the core of the book is none other than the problem of ranking social outcomes on the basis of their consequences for affected individuals. The importance of the exercise cannot be overstated. The standard methodology underlying policy design involves optimizing an objective function subject to constraints. Hence, understanding what we seek to optimize is of paramount importance. The moral values this objective function embodies, the positive assumptions it incorporates, and the conception of the individual it relies on, are all crucial and influential parameters of policy design. Adler's principal contribution is to take the reader on a journey through all the aspects of the problem, while delivering soundly argued opinions along the way.

The exercise of ranking social alternatives for practical purposes is fraught with difficulties and challenges. Although Adler chooses one complete path among many, he is careful to be fair to the alternatives he discards along his way. He reviews a broad array of dissenting opinions on each subject he touches. Moreover, he treats each topic with formal and analytical rigor informed with philosophical insight. In that respect, one source of value for Adler's book is that it serves as a survey. It will acquaint the reader with many facets of the problem, independently of whether the reader chooses to endorse Adler's views.

Primarily, though, the book's main contribution is to bring together two separate schools of thought that have been developing in parallel, and seamlessly merge them into a single account. The book offers insights for the economist, in particular the social choice theorist, who seeks to inform his approach with philosophical perspective. It will help him broaden his understanding of the welfarist foundations of economic theory and to better appreciate the moral justification of the axioms he regularly appeals to.

For the philosopher, the book offers a formal methodology that expands the relevance of the discipline and enables it to arrive at sharper conclusions with policy implications. Adler performs a normative exercise that is valid in a variety of contexts. Consequently, the criterion he delivers can be readily applied to address a broad array of issues. Adler does not merely suggest that the study of normative ethics matters for policy. He identifies a channel through which ethical theories may influence policy. 
There is also, of course, the matter of the particular view that Adler is advocating. Precisely because of the comprehensiveness of his argument and his effort to construct it from the bottom up, Adler is exposing himself to many sources of criticism. This is an inevitable trade-off. From my perspective there are two important components of Adler's argument that may be points of contention.

First, there is the issue of how an individual is modeled as a decision-maker. Here Adler offers his own account, which diverges somewhat from the mainstream, but in the end draws heavily from expected utility theory. The capacity of individuals to make rational and informed decisions when faced with uncertainty is of pivotal importance for Adler's thesis. This capacity is reflected in the individual utility functions and the information they convey. Nonetheless, it seems to me that much of what Adler argues for would carry through even under alternative assumptions about the informational basis of the aggregation exercise (see d'Aspermont and Gevers 2002).

Second, Adler adopts the premise that utilities are interpersonally comparable. There has been a resurgence of research into Arrovian social choice, which is based on purely ordinal and non-interpersonally comparable utilities. This has produced an interesting array of solutions (see Fleurbaey and Maniquet 2011, for a recent survey of this literature). This literature poses two difficulties for Adler's argument. To begin with, it offers a theory of social welfare functions which relies on weaker assumptions than the ones Adler makes. Even if the assumptions on which Adler establishes his argument are reasonable, it must be pointed out that there exists an alternative theory of the social welfare function that relies on weaker ones. Moreover, in this literature the lexi-min criterion features prominently. The axioms that endorse it are justified by principles analogous to the ones Adler adopts.

The crux of the matter is that, in the Arrovian framework, abiding by a mild prioritarian principle, along with other principles that take no stance on the issue of redistribution, produces egalitarianism. Without interpersonal comparability of utilities, and in the absence of any other axiom that encompasses redistributive concerns, a PigouDalton principle appropriately construed to accommodate the Arrovian framework has enough bite to induce an infinite aversion to inequality. This illustrates the fact that much of Adler's defense of prioritarianism hinges on the assumption of the interpersonal comparability of utilities. 
In spite of these remarks, Adler offers an authoritative argument in favor of prioritarianism. I believe it is fair to say that although he does not write the final word on many of the issues he discusses, he nonetheless offers an invaluable contribution to an ongoing dialogue that it is critically important to sustain and nourish.

\section{REFERENCES}

d'Aspremont, Claude, and Louis Gevers. 2002. Social welfare functions and interpersonal comparability of utilities. In Handbook of social choice and welfare, vol. I, eds. Kenneth Arrow, Amartya Sen, Kotaro Suzumura. Amsterdam: NorthHolland, 459-541.

Fleurbaey, Marc, and François Maniquet. 2011. A theory of fairness and social welfare. Cambridge (UK): Cambridge University Press.

Moulin, Herve. 1988. Axioms of cooperative decision making. Cambridge (UK): Cambridge University Press.

Efthymios Athanasiou is assistant professor of economics at the New Economic School in Moscow, Russia. Between 2009 and 2011 he held the Herbert Simon Fellowship in Scientific Philosophy at Carnegie Mellon University. His research interests include ethics, social choice, and mechanism design.

Contact e-mail: <timos.ath@gmail.com> 